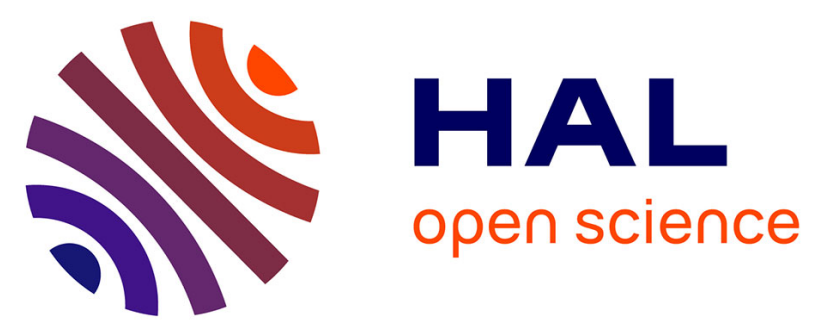

\title{
Book review - A Spirited Exchange. The Wine and Brandy Trade Between France and the Dutch Republic in Its Atlantic Framework, 1600-1650, by Henriette de Bruyn Kops, Leiden-Boston
}

Mathieu Grenet

\section{To cite this version:}

Mathieu Grenet. Book review - A Spirited Exchange. The Wine and Brandy Trade Between France and the Dutch Republic in Its Atlantic Framework, 1600-1650, by Henriette de Bruyn Kops, LeidenBoston. European Review of History / Revue européenne d'histoire, 2009, 16 (4), pp.597-598. 10.1080/13507480903063928 . hal-02303321

\section{HAL Id: hal-02303321 \\ https://hal-univ-tlse2.archives-ouvertes.fr/hal-02303321}

Submitted on 21 Jan 2021

HAL is a multi-disciplinary open access archive for the deposit and dissemination of scientific research documents, whether they are published or not. The documents may come from teaching and research institutions in France or abroad, or from public or private research centers.
L'archive ouverte pluridisciplinaire HAL, est destinée au dépôt et à la diffusion de documents scientifiques de niveau recherche, publiés ou non, émanant des établissements d'enseignement et de recherche français ou étrangers, des laboratoires publics ou privés. 
A Spirited Exchange. The Wine and Brandy Trade Between France and the Dutch Republic in Its Atlantic Framework, 1600-1650, by Henriette de Bruyn Kops, Leiden-Boston, Brill, 2007, XIII + 371 pp., €99/\$139, ISBN 9789004160743 (hbk)

Il existe au moins trois manières de lire l'étude qu'Henriette de Bruyn Kops consacre au commerce du vin et de l'eau-de-vie entre Nantes et Rotterdam dans la première moitié du $\mathrm{XVII}^{\mathrm{e}}$ siècle. La première consiste à y voir une monographie venue pallier un chapitre méconnu de l'histoire des échanges entre la France et la République hollandaise. La seconde s'attarde sur la critique radicale qu'adresse l'auteure à l'historiographie de l'"âge d'or" néerlandais, et sa réévaluation de l'importance relative des différents secteurs du commerce maritime hollandais. La troisième, enfin, se focalise sur le modèle qu'elle propose d'une interdépendance économique et commerciale à l'échelle de l'espace atlantique. Pourquoi ces trois lectures plutôt qu'une seule ? Simplement parce que si le plus grand mérite du travail d'Henriette de Bruyn Kops est d'explorer avec application chacun de ces thèmes, son plus grand défaut est de ne pas parvenir à les lier de manière convaincante.

L'ouvrage est divisé en une introduction et six chapitres, eux-mêmes subdivisés en une myriade de petites sections qui rendent la lecture excessivement morcelée. Le premier chapitre traite de la présence hollandaise à Nantes dans la première moitié du XVII ${ }^{\mathrm{e}}$ siècle : s'installant sur les bords de la Loire à partir des années 1620, les marchands de Rotterdam viennent s'y approvisionner en vins de faible prix et de mauvaise qualité, qu'ils "corrigent" pour en faire une eau-de-vie qu'ils réexportent alors vers Amsterdam. À une étude méticuleuse de la chronologie de l'arrivée des Hollandais à Nantes, l'auteure ajoute un intéressante analyse de la relation parfois conflictuelle qu'entretiennent marchands locaux et

Mathieu Grenet, c/o EUI, Villa Schifanoia, via Boccaccio 121, 50133 Firenze, Italia, +39 3488255065, mathieu.grenet@eui.eu 
étrangers, et montre comment ces derniers parviennent fréquemment à obtenir gain de cause en s'adressant aux autorités nantaises, et parfois même parisiennes. Dans le second chapitre, de Bruyn Kops propose un tableau détaillé du milieu des marchands de vin de Rotterdam, qu'elle répartit en trois catégories (grands négociants internationaux, acheteurs et grossistes, et petits revendeurs locaux) selon leur importance économique et leur rayon d'action. Afin de démontrer le fort degré d'intégration commerciale qui lie les différents réseaux du grand et du petit commerce des vins, l'auteure se penche alors sur leur fonctionnement entre la France et la Hollande. Malgré l'ambition affichée d'une telle analyse, celle-ci se révèle au final assez décevante, et l'examen de certains aspects essentiels de la question (les stratégies familiales, l'importance de la parentèle, etc.) n'est qu'ébauché.

Les deux chapitres suivants sont consacrés à une minutieuse étude du commerce $d u$ vin entre Nantes et Rotterdam, deux ports dont l'intérêt réside précisément dans la place secondaire qu'ils occupent dans une hiérarchie commerciale dominée par Amsterdam et Bordeaux - cette dernière ville se taille ainsi la part du lion dans un commerce d'exportation des alcools français vers la Hollande auquel Nantes ne participe qu'à hauteur de $10 \%$. C'est pourtant dans cette partie de l'ouvrage qu'Henriette de Bruyn Kops se montre le plus à son aise, notamment par son habileté à manier des données pour le moins lacunaires. En témoigne le tour de force que constitue la reconstruction qu'elle propose des grandes tendances du commerce nantais à partir du seul registre portuaire de 1631 , l'unique à nous être parvenu. De manière peut-être plus anecdotique, elle consacre aussi des pages remarquables à des sujets aussi divers que les différents types de tonneaux ou le système de taxation du vin. Mais audelà de ces beaux morceaux de ce que Michel Morineau appellerait "une histoire économique vraie", l'auteure étaye et développe dans ces chapitres ce qui constitue sans doute la principale contribution de son ouvrage. Partant de l'exemple nantais, elle démontre en effet que le commerce côtier le long de la façade atlantique de l'Europe constitue un secteur 
fondamental du commerce maritime hollandais au XVII ${ }^{\mathrm{e}}$ siècle. Bien que moins "glamorous" (p. 304) que les grands négoces baltique et transocéaniques tant célébrés par l'historiographie de l'âge d'or hollandais, il les égale pourtant - voire les dépasse - en terme de valeur économique. Mieux, il est à la source de l'accumulation de capital qui permet de financer d'autres entreprises commerciales de plus grande ampleur : plus qu'à rétablir une hiérarchie entre les trois grandes branches du commerce maritime hollandais, de Bruyn Kops vise donc à démontrer leur essentielle complémentarité comme leur profonde interdépendance. Elle y parvient brillamment, et ouvre du même coup la voie à une relecture en profondeur des travaux d'historiens aussi éminents que Jan de Vries, Ad Van der Woude et Jonathan Israel.

De Bruyn Kops aurait sans doute pu (et dû) s'en tenir à cette démonstration. Elle fait pourtant le choix assez inexplicable de bifurquer sur une étude des réseaux commerciaux sépharades dans l'espace atlantique. La quasi absence de lien entre les marchands sépharades et le commerce des vins et spiritueux ne constitue pas le moindre des problèmes de ce cinquième chapitre. Une chose est en effet d'affirmer que Nantes constitue un lieu d'intersection des réseaux commerciaux hollandais et juifs (p. 261), et une autre est d'analyser les différentes modalités selon lesquelles opère cette "intersection". Au lieu de cela, de Bruyn Kops brosse le tableau quelque peu irénique d'un grand réseau marchand atlantique, où Juifs, Catholique, Protestants et Nouveaux Chrétiens coopèrent (p. 298). Quant à 1'hypothèse plusieurs fois formulée d'un lien entre commerce des vins nantais et contrebande d'argent espagnol (pp. 276, 295), elle présente certes l'avantage d'être séduisante, mais aussi l'inconvénient notoire de ne reposer sur aucune preuve tangible.

Le dernier chapitre de l'ouvrage se présente comme une tentative de lecture "globale" de ceux qui précèdent. L'auteure s'y essaie alors à un exercice intéressant, qui consiste à resituer son cas nantais dans le contexte plus vaste non seulement du commerce côtier, mais aussi d'une économie qu'elle qualifie de "Dutch-Atlantic". S'il finit par aboutir sur la 
proposition originale d'un modèle d'analyse économique inspiré du Rubik's Cube (!), ce chapitre s'organise autour d'une série de thèmes disparates dont l'articulation semble pour le moins artificielle.

S'il en était besoin, l'absence d'une conclusion suffit à illustrer la principale faiblesse d'un ouvrage qui constitue néanmoins un très bon exemple de ce qu'une approche binationale et comparative peut apporter à l'histoire commerciale.

Mathieu Grenet

European University Institute

mathieu.grenet@eui.eu 